\title{
Brain-Computer Interface Using Near-Infrared Spectroscopy for Rehabilitation
}

\author{
Kazuki Yanagisawa, Hitoshi Tsunashima and Kaoru Sakatani \\ Nihon University \\ Japan
}

\section{Introduction}

Currently, the Brain Computer Interface $(\mathrm{BCI})$ is being studied vigorously. BCI extracts thoughts in the human brain as cranial nerve information and uses the information as inputs to control machinery and equipment. Fig. 1 describes schematic BCI system. If this system enables operating machinery and equipment directly from cranial nerve information without the subject moving his or her hands and feet, it can be applied to care-taking robots and rehabilitation for physically handicapped individuals.

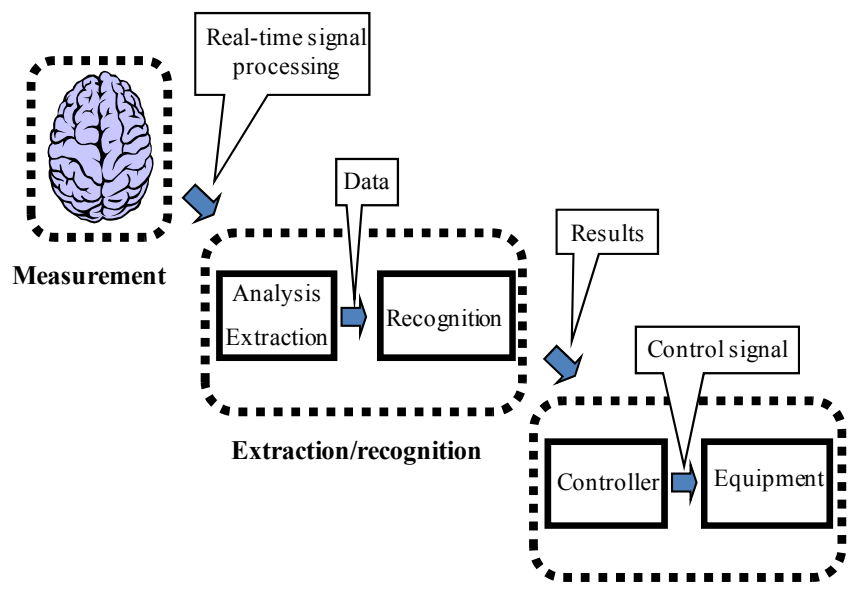

Control

Fig. 1. Schematic of BCI system

BCI systems can be divided into two forms. The invasive form reads cranial nerve information using electrodes embedded directly into the brain. The non-invasive form reads cranial nerve activity from the surface of the head using near infrared spectroscopy (NIRS) or electroencephalography (EEG). as an example of invasive form, Donoghue LR. et al. extracted nerve activity of primary motor area and controlled a robot hand and mouse cursor (Hochberg LR et. Al, 2006). Though the invasive form has high signal accuracy, it imposes a heavy load on the user (e.g., surgery and infections after surgery). 
Therefore, the non-invasive form has a wider applicable range. In a study on the noninvasive form, Pfurtscheller have developed BCI that physically handicapped individuals can control a character of the virtual reality (Pfurtscheller G. et. Al, 2006), electricaldriven hand (Pfurtscheller G. et. al, 2000) and functional electrical stimulation (FES) ( Pfurtscheller G., 2003). Vaughan TM et al. have developed in prototype systems for everyday use in people's homes for locked-in patients (Vaughan TM, 2006). Many other studies of BCI have used EEG. However, EEG has low spatial resolution and it is vulnerable to electrical noise. In contrast, NIRS imposes fewer restrictions on body movement than EEG does and is more resistant to electric noise, so the load imposed on the user is less and electronic devices have no influence. And, NIRS has high space resolution. Therefore, the present study focuses on BCI that uses NIRS.

In an earlier study, Nagaoka et al. developed a NIRS-BCI rehabilitation system (Nagaoka T, 2010). In their study, electric stimuli corresponding to cranial nerve information are applied to the user's biceps brachii muscle by setting a threshold on signals measured from NIRS, in order to cause the elbow joint to move. But, Signals measured by NIRS are unstable because they include signals components that are irrelevant to the subject (e.g., noise of measuring instruments, heartbeat, and respiration). Moreover, a processing method for NIRS signals has not yet been established. For these reasons, it is difficult obtain a high identification rate by merely setting a simple threshold on NIRS signals. And this BCI system lacks versatility, because it is large system using multi-channel NIRS equipment.

In this study, we propose a new detection method that uses oxy- $\mathrm{Hb}$ and its differential as indexes for application to the NIRS-BCI rehabilitation system, detecting brain activity from the data measured using NIRS. First, we develop a BCI system to control robot arm using NIRS, and confirm that NIRS-BCI system can control machine and device. Next, we apply NIRS-BCI system developed for rehabilitation.

\section{Near-infrared spectroscopy (NIRS)}

Using near-infrared rays, NIRS non-invasively measures changes in cerebral blood flow. The principle of measurement was developed by Jöbsis (1977), based on the measurement of hemoglobin oxygenation in the cerebral blood flow.

In uniformly distributed tissue, incident light is attenuated by absorption and scattering. The following expression, a modified Lambert-Beer law, was therefore used:

$$
A b s=-\log \left(I_{\text {out }} / I_{\text {in }}\right)=\varepsilon \bar{l} C+S .
$$

Here, $I_{\text {in }}$ is the irradiated quantity of light; $I_{\text {out }}$ is the detected quantity of light; $\varepsilon$ is the absorption coefficient; $C$ is the concentration; $\bar{l}$ is the averaged path length; and $S$ is the scattering term.

If it is assumed that no scattering changes in brain tissue occur during activation of the brain, the change in absorption across the activation can be expressed by the following expression:

$$
\Delta A b s=-\log \left(\Delta I_{\text {out }} / \Delta I_{\text {in }}\right)=\varepsilon \bar{l} \Delta C\left(\Delta X_{\text {oxy }}, \Delta X_{\text {deoxy }}\right) .
$$


Furthermore, if the change in concentration $(\Delta C)$ is assumed to be proportional to the changes in oxygenated hemoglobin $\left(\Delta X_{o x y}\right)$ and deoxygenated hemoglobin $\left(\Delta X_{\text {deoxy }}\right)$, the following relational expression can be obtained:

$$
\Delta A b s\left(\lambda_{i}\right)=\bar{l}\left[\varepsilon_{\text {oxy }}\left(\lambda_{i}\right) \Delta X_{\text {oxy }}+\varepsilon_{\text {deoxy }}\left(\lambda_{i}\right) \Delta X_{\text {deoxy }}\right] .
$$

The absorption coefficients of oxygenated hemoglobin and deoxygenated hemoglobin at each wavelength, $\varepsilon_{o x y}\left(\lambda_{i}\right)$ and $\varepsilon_{\text {deoxy }}\left(\lambda_{i}\right)$, are known. As a result, $\bar{l} \Delta X_{\text {oxy }}$ and $\bar{l} \Delta X_{\text {deoxy }}$ can be obtained by performing measurements with near-infrared rays of two different wavelengths and solving simultaneous equations for Equation 3. However, the physical quantity obtained here is the product of the change in concentration and the averaged path length. In general, this averaged path length $\bar{l}$ varies greatly from one individual to another, and from one part of the brain to another. Caution must therefore be exercised in evaluating the results.

In general, changes in oxygenated hemoglobin and deoxygenated hemoglobin when the brain is activated and restored to the original state exhibit the trend illustrated in Figure 2 (Huettel, 2004).

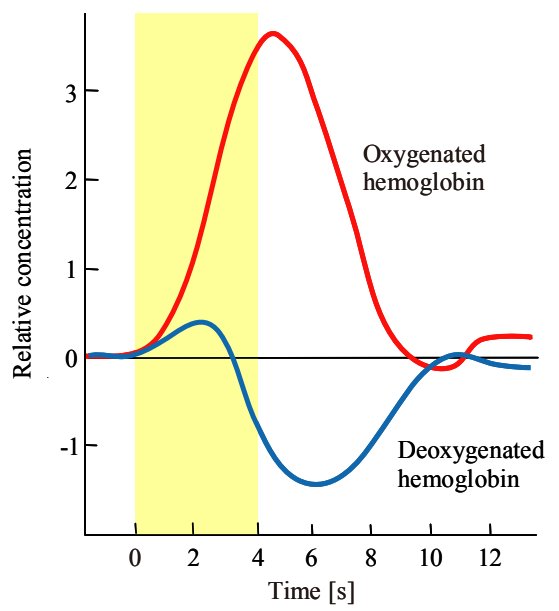

Fig. 2. Schematic of changes in hemoglobin concentration due to neural activity

\section{Brain-computer interface using NIRS}

Figure 3 depicts a robot control system that uses NIRS-BCI. This system is composed of a cerebral function measurement section, a feature extraction and recognition section, and a device control section. In the cerebral function measurement section, the subject's oxy- $\mathrm{Hb}$ is measured using a multichannel NIRS instrument, OMM-3000, made by Shimadzu Corporation, Japan. The system operated at three different wavelengths of 780,805,830nm. In the feature extraction and recognition section, the threshold is obtained by analyzing the original signal of oxy- $\mathrm{Hb}$ that was measured. When oxy- $\mathrm{Hb}$ after analysis exceeds the threshold obtained in the feature extraction and recognition section, the on signal is sent to the device control section in order to enable rotation of the joint of the robot arm (MR-999; Elekit, Japan). 

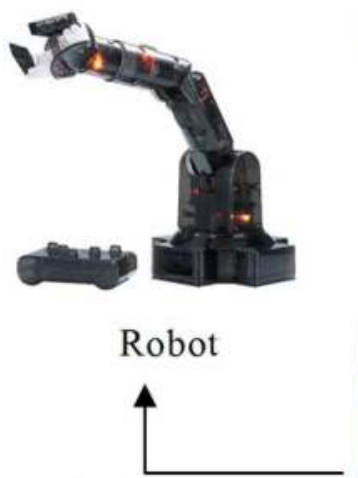

$\mathrm{ON} / \mathrm{OFF}$

Transmission of

a signal

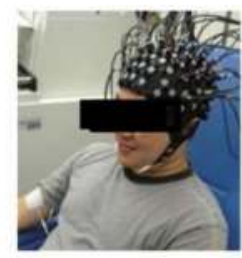

Subject

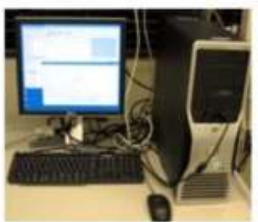

Extraction/recognition

Fig. 3. BCI robot control system

Subjects were conducted using the robot arm control system depicted in Fig. 1. Five trials were carried out, with each trial consisting of $10 \mathrm{sec}$ of pre-task rest, $30 \mathrm{sec}$ of task, and 10sec of post-task rest. The first two trials were defined as the learning stage, where the feature extraction and recognition section learned the fluctuation pattern of the subject's oxy- $\mathrm{Hb}$ without moving the robot. In the third and subsequent trials, the robot's arm was rotated according to the learned pattern corresponding with oxy-Hb. The subject was instructed to perform grasping during tasks and instructed to rest during the rest time. The motor area was selected as the measurement site.

Optical fibers were arranged in $4 \times 4$ matrices on the right and left sides to perform measurement, with a total of 48 channels (Fig.4). The subjects were two healthy male

volunteers in their twenties. Prior informed consent was obtained from all subjects, in an effort to ensure full consideration of their safety and protection of their human rights.

In the detection method that used a simple threshold, the moving average was obtained. The threshold was set to $20 \%$ of the maximum oxy- $\mathrm{Hb}$ during the first two trials; detection was made in the third and subsequent trials, and an on state was judged when oxy- $\mathrm{Hb}$ exceeded the threshold.

Changes in concentration of oxy- $\mathrm{Hb}$ and the detection results for grasping tasks are depicted in Fig.5. ON detections were made in tasks from the first to second trials. OFF decisions in rest were wrong. ON and OFF detections alternated within a short time, indicating instability. 


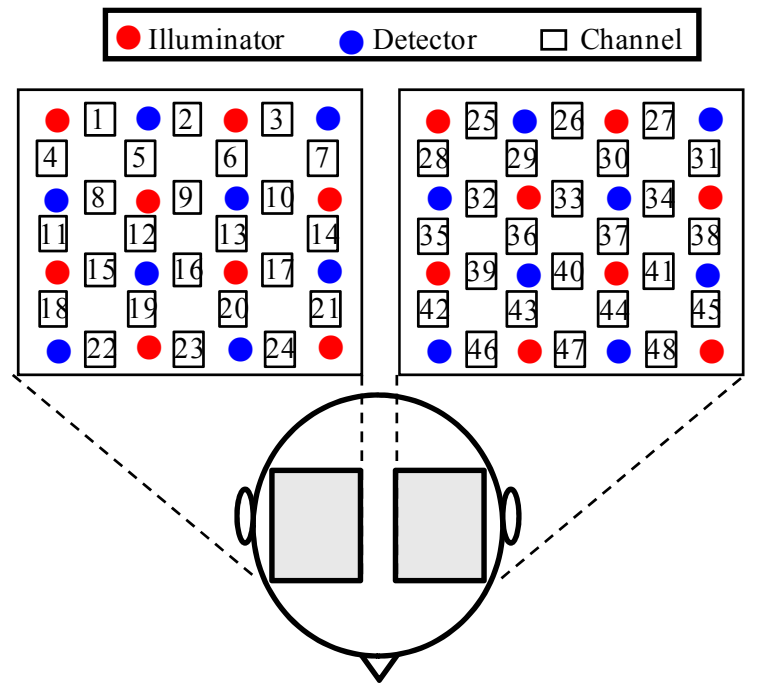

Fig. 4. Position of optical fibers and channels for recording NIRS signals (grasping tasks: matrix, 48 channels)

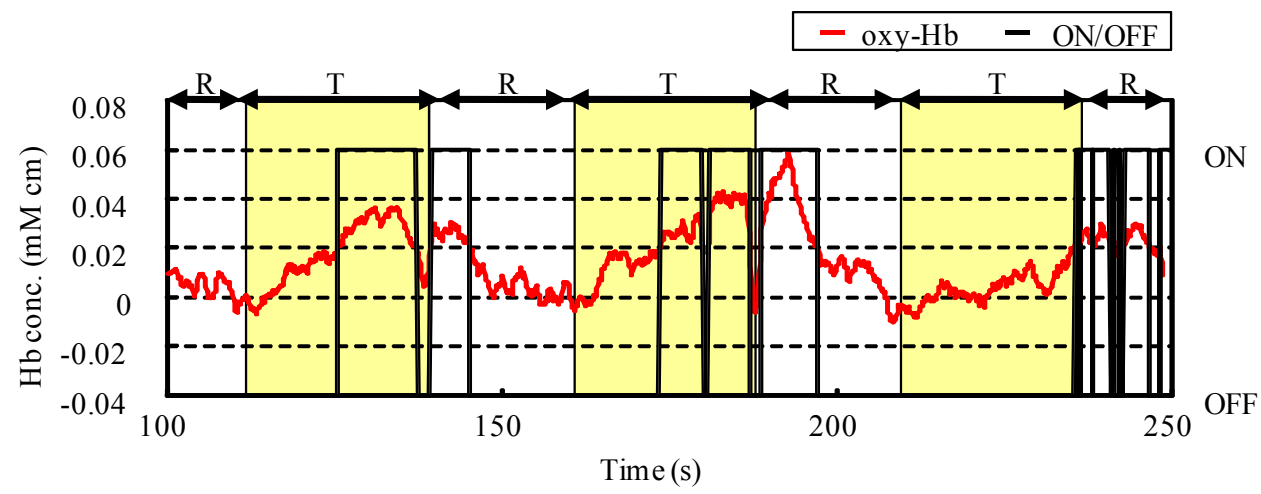

Fig. 5. Result of ON/OFF decision using conventional method

\section{Signal processing method for NIRS-BCI}

\subsection{Detection method of brain activity}

NIRS signals also include signals that are not related to brain activity (e.g., measuring device noise, influences of respiration, and fluctuations of blood pressure). Therefore, to evaluate brain activity in detail, it is necessary to remove these extraneous signals. For this purpose, we selected the channels that have a good $\mathrm{S} / \mathrm{N}$ ratio and exhibit remarkable task-related fluctuations (44 channels (contralateral motor cortex) in this case), and subjected the NIRS signals obtained from them to magnetic resonance angiogram analysis (MRA) (Kojima et. al, 2006, Yanagisawa et. al, 2009, Tsunashima et. al, 2009) through discrete wavelet transformation to decompose and reconfigure the signals. 
Figure 6 presents the MRA results for oxy- $\mathrm{Hb}$ in the 44 channels, where task-related changes were remarkable. The components of the very short frequency bands of $d_{1}$ and $d_{2}$ correspond to the measurement noises. The components of $\mathrm{d}_{3}$ and $\mathrm{d}_{4}$ which contains the signal period from 3 to 4 seconds correspond to the breathing. The components of $d_{5}$ and $d_{6}$ include changes of blood pressure (Elwell C.E et al. 1999). The trend of the whole experiment was extracted on the approximated component $\left(\mathrm{a}_{10}\right)$. Because the interval of repetition of tasks and rests was $50 \mathrm{sec}$, the $\mathrm{d}_{8}$ component was the central component of taskrelated changes. Therefore, signals were reconstructed by adding the $d_{7}, d_{8}, d_{9}$ and $d_{10}$ components.

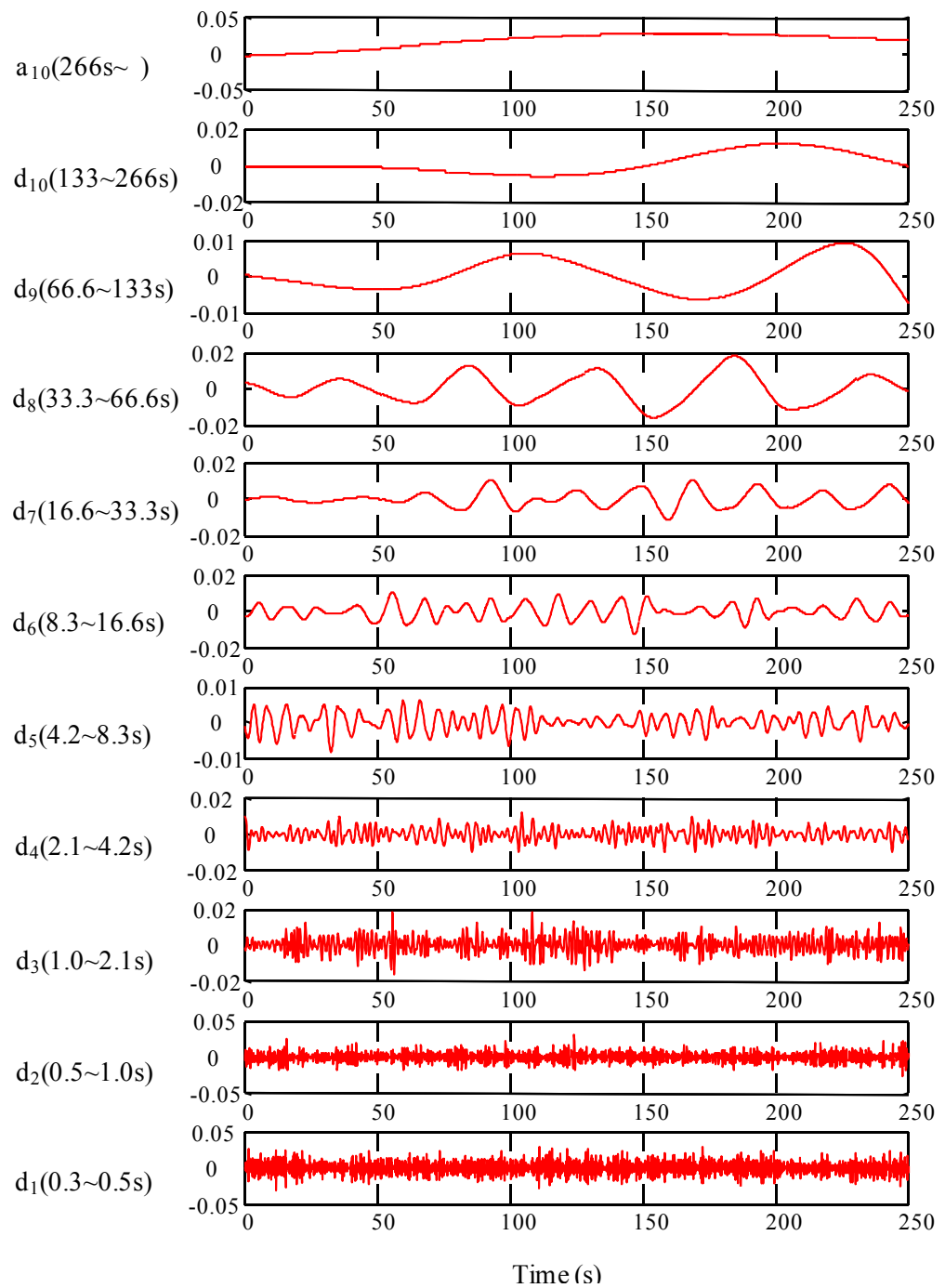

Fig. 6. Decomposition of NIRS signal (channel 44) 
Reconstructed signals from channel 44 are illustrated in Figure 7. Results revealed that oxygenated hemoglobin increased and the brain was activated during grasping tasks.

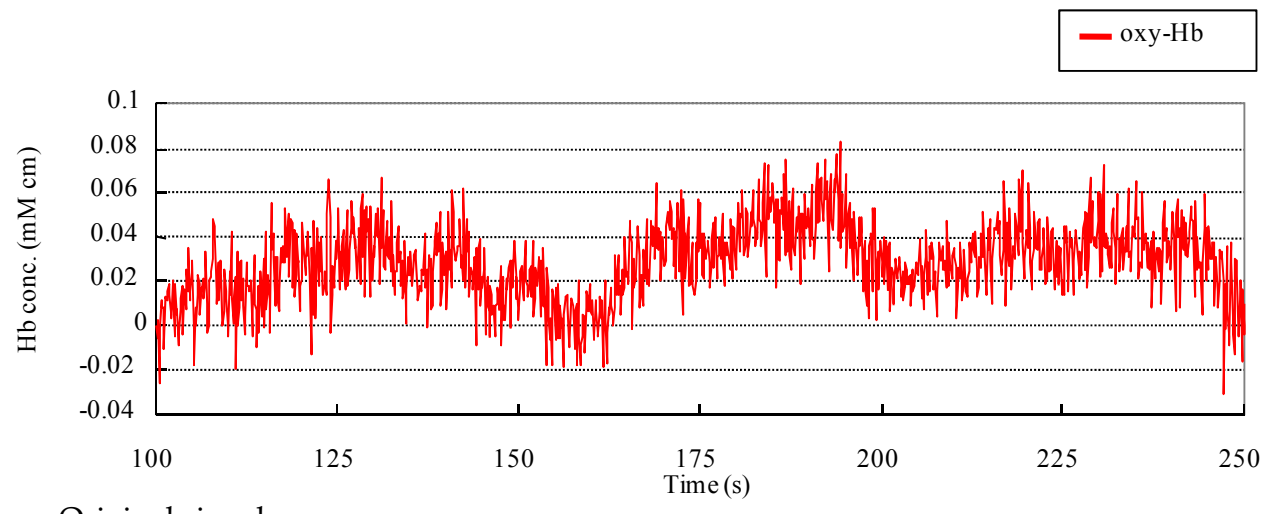

a. Original signal

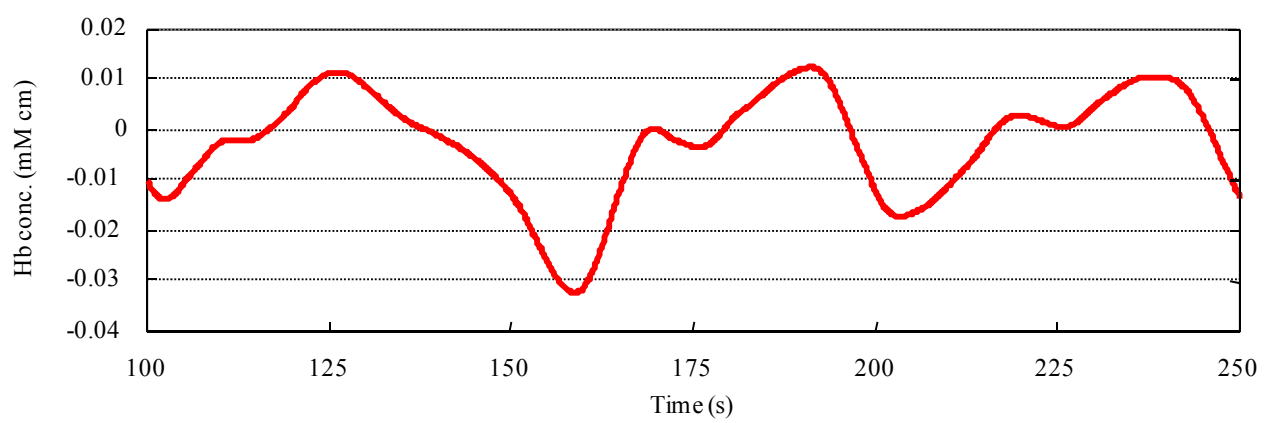

b. Reconstructed NIRS signal

Fig. 7. Comparison of original signal and reconstructed signal (channel44)

We propose the detection method of brain activity. NIRS can measure changes in concentrations of oxy- $\mathrm{Hb}$ and deoxy- $\mathrm{Hb}$, as well as the total- $\mathrm{Hb}$ signal (the sum of these two values). In this study, we focus on the oxy- $\mathrm{Hb}$ signal because changes in concentration of oxy- $\mathrm{Hb}$ are highly correlated with the regional cerebral blood flow (rCBF) (Sakatani et, al, 2007), and an increase in rCBF reflects an increase in neural activity (Jueptner, et. al, 1195) Furthermore, we evaluate brain activity using two indexes, oxy- $\mathrm{Hb}$ and its differential, because the differential of oxy-Hb is correlated with the workload of the task (Shimizu T. et. al, 2009).

Common fluctuations of oxy-Hb and its differential are depicted in Fig. 8 (a). As the detection method, we propose that oxy- $\mathrm{Hb}$ be taken on the horizontal axis and the differential of oxy- $\mathrm{Hb}$ on the vertical axis, and detection be made from the area of its trajectory. The trajectory of the activity in Fig. 8(a) is depicted in Fig. 8(b). When the trajectory passes through the red zone in Fig. 8(b), it can be assumed that brain activity is occurring. 


$$
- \text { oxy-Hb }-\mathrm{d}(\mathrm{oxy}-\mathrm{Hb}) / \mathrm{dt}
$$

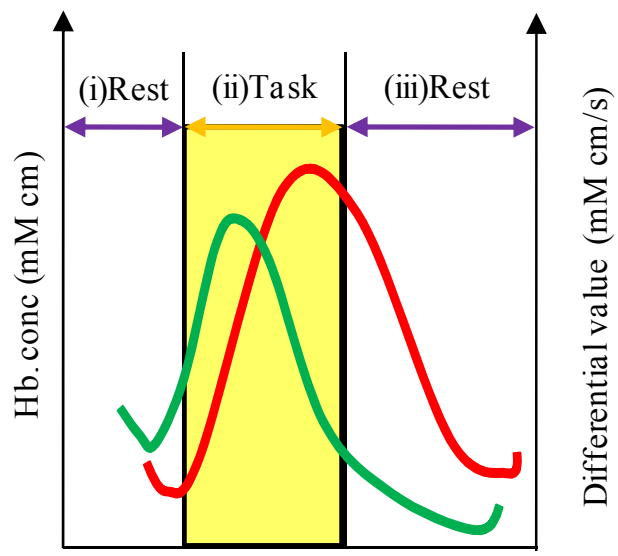

Time $(s)$

a. Change of oxy-Hb and differential value

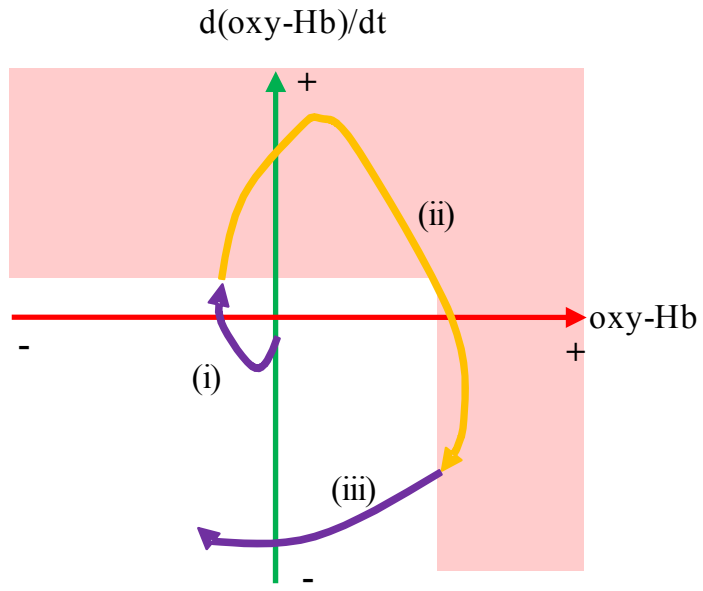

$$
\text { - Task }- \text { Rest }
$$

Activation area

b. Trajectory of oxy-Hb and $\mathrm{d}(\mathrm{oxy}-\mathrm{Hb}) / \mathrm{dt}$

Fig. 8. Relations between oxy-Hb and differential

About grasping tasks, we selected the channels that have a good $\mathrm{S} / \mathrm{N}$ ratio and exhibit remarkable task-related fluctuations (44 channels (contralateral motor cortex) in this case), and subjected the NIRS signals obtained from them to MRA through discrete wavelet transformation to decompose and reconfigure the signals.

Figure 9 depicts the trajectory with oxy- $\mathrm{Hb}$ on the horizontal axis and the differential of oxy$\mathrm{Hb}$ on the vertical axis using the signals from grasping tasks as reconfigured. As with the 

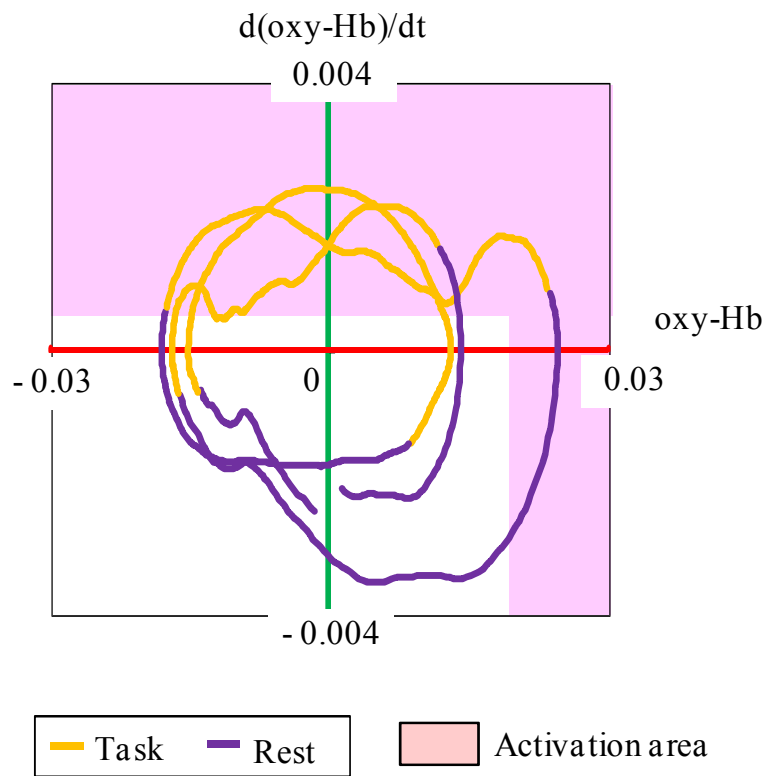

Fig. 9. Trajectory of oxy- $\mathrm{Hb}$ and differential value (grasping task)

trajectory depicted in Fig. 8 (b), the trajectory passes through the red zone, where brain activity is assumed to be occurring during the task.

The area where both oxy-Hb and its differential increase (first quadrant) is assumed to be the area where brain activity is occurring. When the differential of oxy- $\mathrm{Hb}$ is large, it can be assumed that brain activity is occurring even if the value of oxy- $\mathrm{Hb}$ is small. When the activity level is low, such as during rest time, the trajectory tends to draw a circle of the area of activity around the datum point. To avoid judging the trajectory of the signals that pass through the area around the datum point as a sign of activity, a threshold will be set on the differential of oxy-Hb. However, the differential of oxy-Hb does not always continue to increase when activity is occurring, so we assume that activity is also occurring when oxy$\mathrm{Hb}$ is above a threshold level. Furthermore, when the trajectory does not stay in the area for more than a specified period (2.0s), we assume that it is an influence of artifact and that no activity is occurring.

\subsection{Detection result}

Figure 10 presents the detection results with the proposed detection method applied. Comparison with the results of the conventional detection method presented in Fig. 35confirmed the ON detection during all tasks. Furthermore, the threshold was set on the value of oxy- $\mathrm{Hb}$ alone in the conventional detection method, so it was impossible to make the ON detection during the first half of a task with a low level of oxy- $\mathrm{Hb}$, and the detection result was delayed even if relevant brain activity could be confirmed. The proposed detection method, however, reduced the delay due to using the differential of oxy- $\mathrm{Hb}$.The proposed detection method did enable highly accurate detection of many subjects, compared with the conventional detection method. 


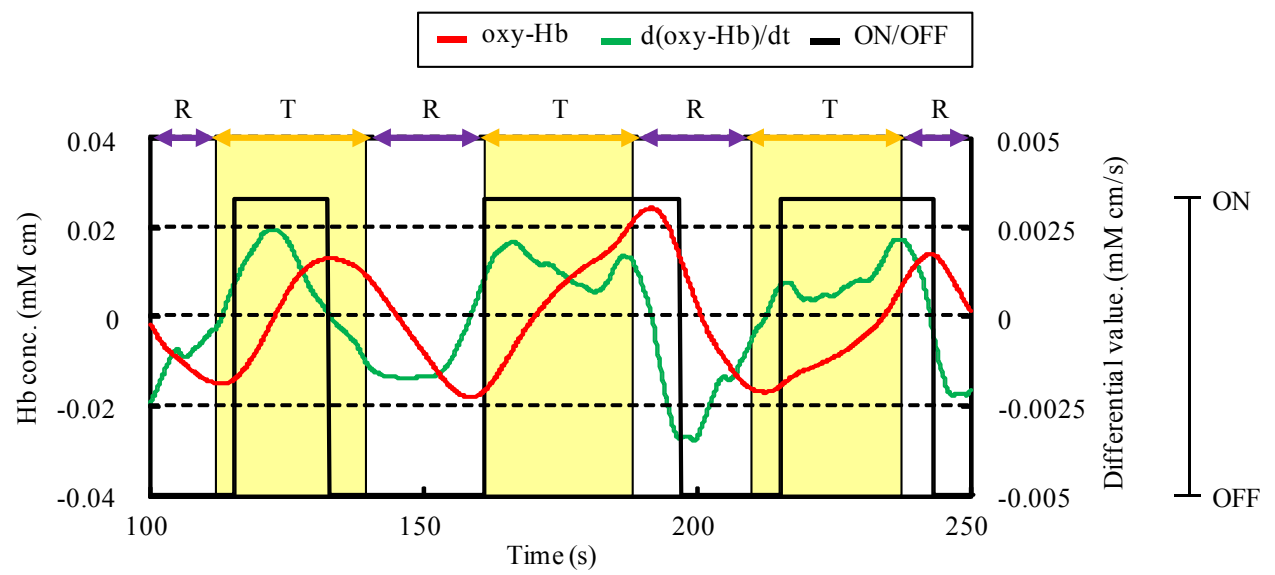

Fig. 10. Result of ON/OFF decision using proposed method (grasping task)

On the same condition, the subject was instructed to imagine grasping without moving the hand during the tasks. The detection of brain activity during imagined grasping tasks using the proposed detection method is presented in Fig. 11. Also during imagined grasping tasks, as in actual grasping tasks in Fig. 10, the ON detection was confirmed during all tasks. Furthermore, a correct detection could be made with little time delay, due to using the differential of oxy-Hb. These results confirm that the proposed detection method is valid for imagined grasping tasks as well.

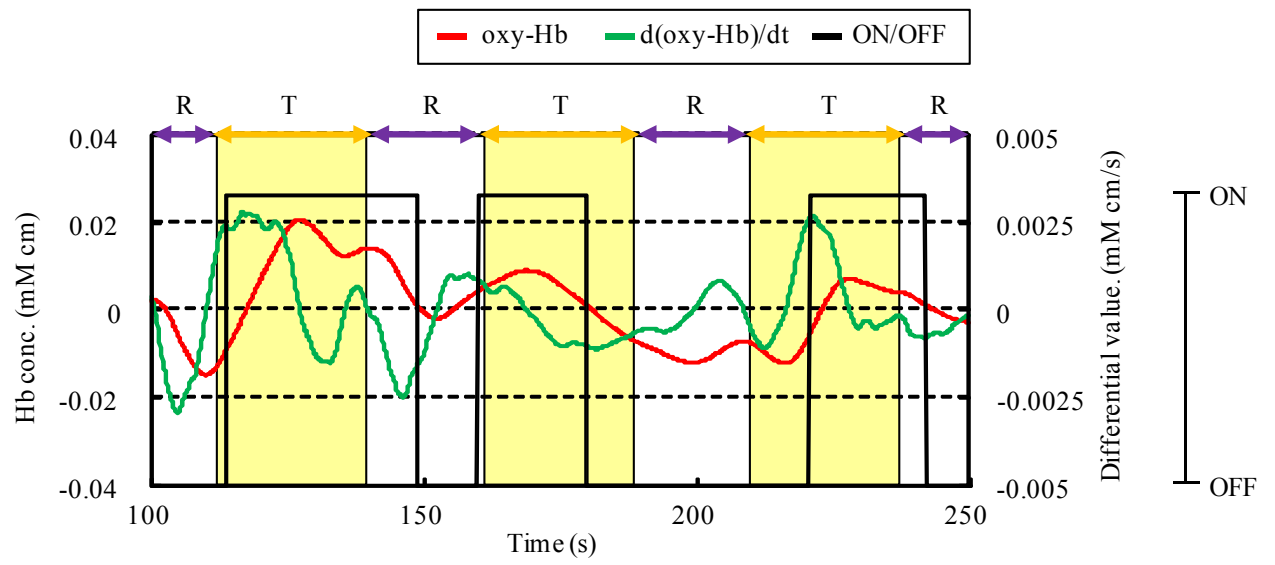

Fig. 11. Result of ON/OFF decision using proposed method (imagined grasping tasks)

\section{IRS-BCI system for rehabilitation}

\subsection{BCl-rehabilitation system}

In the NIRS-BCI rehabilitation system (Fig.12), the signals measured with NIRS are transferred to the analysis workstation in real time. A threshold is set based on oxy- $\mathrm{Hb}$ 


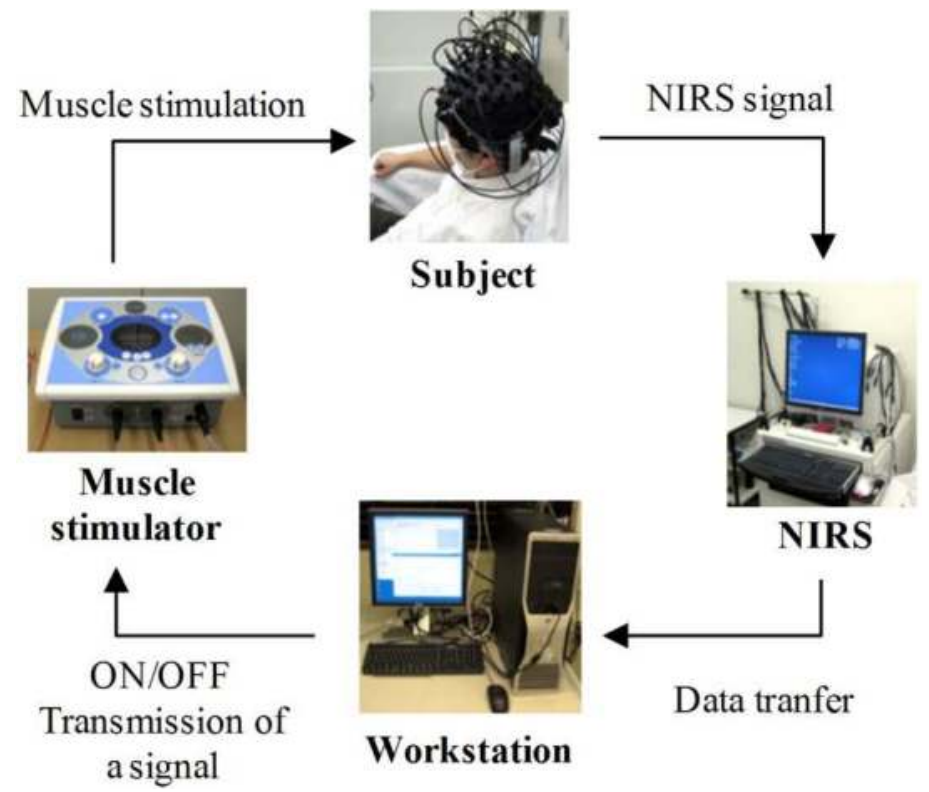

Fig. 12. NIRS-BCI rehabilitation system

signals that are transferred; when the signals exceed the threshold, the percutaneous electrical stimulator gives percutaneous electrical stimulation to the biceps brachii muscle to induce an elbow joint refraction movement. Therefore, even those who cannot move their arms can actually participate in rehabilitation.

\subsection{Detection of brain activity}

We conducted experiments using the NIRS-BCI rehabilitation system depicted in Fig. 12. In the experiment, five cycles are performed: each cycle consists of 10sec of pre-task rest, 30sec of task, and 10sec of post-task rest. The threshold is set during the first two cycles, and muscle stimulation is applied when oxy- $\mathrm{Hb}$ has exceeded the threshold during the third and succeeding cycles.

Two kinds of tasks were set: one to perform actual grasping and the other to imagine grasping. The subject was instructed to perform grasping with right hand during actual grasping tasks, and to imagine grasping without moving the hand during the imagined grasping tasks. The biceps brachii muscles of the left arm were stimulated. In either task, the subject was instructed to rest during rest time.

To consider application of this system for rehabilitating patients with hemiplegia, muscles must be stimulated by detecting brain activity during imagined grasping tasks instead of during actual grasping tasks. However, large differences exist between individuals' brain activity during imagined grasping tasks. Therefore, in this study, we confirm the validity of the proposed detection method by conducting grasping tasks associated with significant brain activity appears remarkably. Furthermore, we will apply the proposed detection method to brain activity during imagined grasping tasks. 
Brain activity in the motor area was measured using NIRS. The measurement device was a near-infrared device made by Shimadzu Corporation (multichannel NIRS instrument, OMM-2001, Shimadzu Corporation, Japan). Figure 13 depicts a scene of the experiment. With $4 \times 4$ probes arranged on the left and right sides, measurement was performed with a total of 48 channels (Fig. 4). Seven healthy males in their twenties were selected as subjects, and informed consent was obtained from them before conducting the experiment after explanation of the experiment purpose.

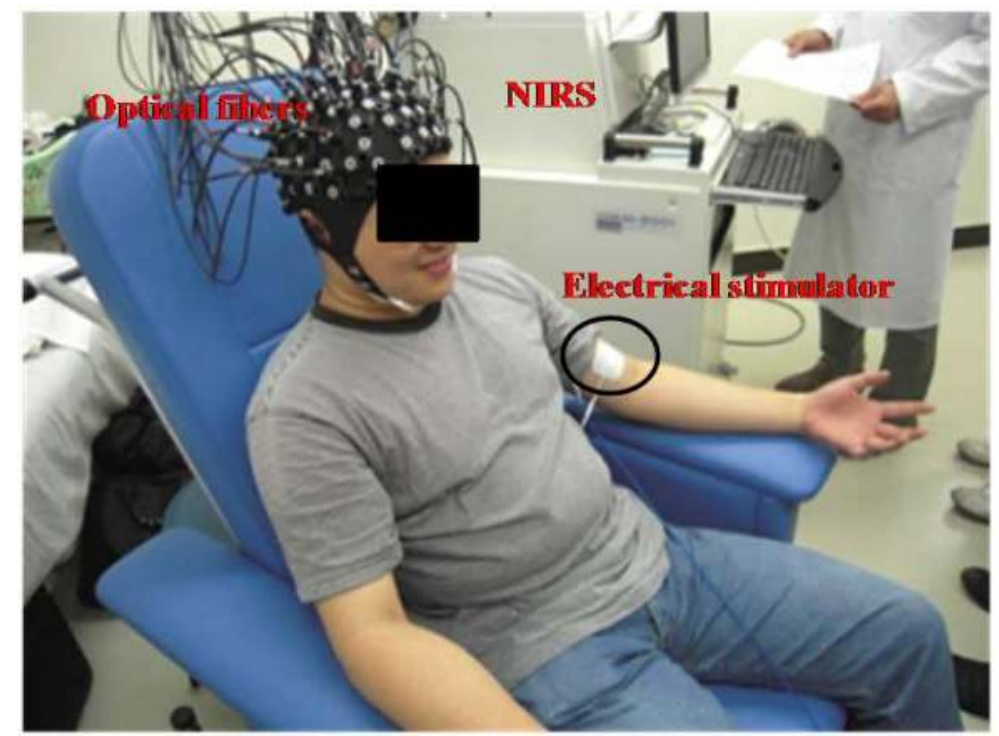

Fig. 13. Scene of the experiment

In the conventional detection method, the z-score was established from the mean value of the first two cycles of the signals measured by NIRS, and the standard deviation and the moving average were obtained. The threshold was set to $20 \%$ of the maximum oxy- $\mathrm{Hb}$ value obtained during the first two cycles; detection was made on the third and succeeding cycles, where the values of oxy- $\mathrm{Hb}$ that exceeded the threshold were judged as $\mathrm{ON}$.

Changes in concentration of oxy- $\mathrm{Hb}$ during grasping tasks and the detection results are presented in Fig. $14 \mathrm{ON}$ detection was observed during the first task; however, it was confirmed that no ON detection was made during the succeeding tasks, even though some changes in oxy-Hb concentration of were detected.

Figure 15 presents the detection results with the proposed detection method applied. Comparison with the results of the conventional detection method presented in Fig. 14 confirmed the ON detection not only during the first task but during all tasks. Furthermore, the threshold was set on the value of oxy- $\mathrm{Hb}$ alone in the conventional detection method, so it was impossible to make the ON detection during the first half of a task with a low level of $\mathrm{oxy}-\mathrm{Hb}$, and the detection result was delayed even if relevant brain activity could be confirmed. The proposed detection method, however, reduced the delay due to using the differential of oxy-Hb. 


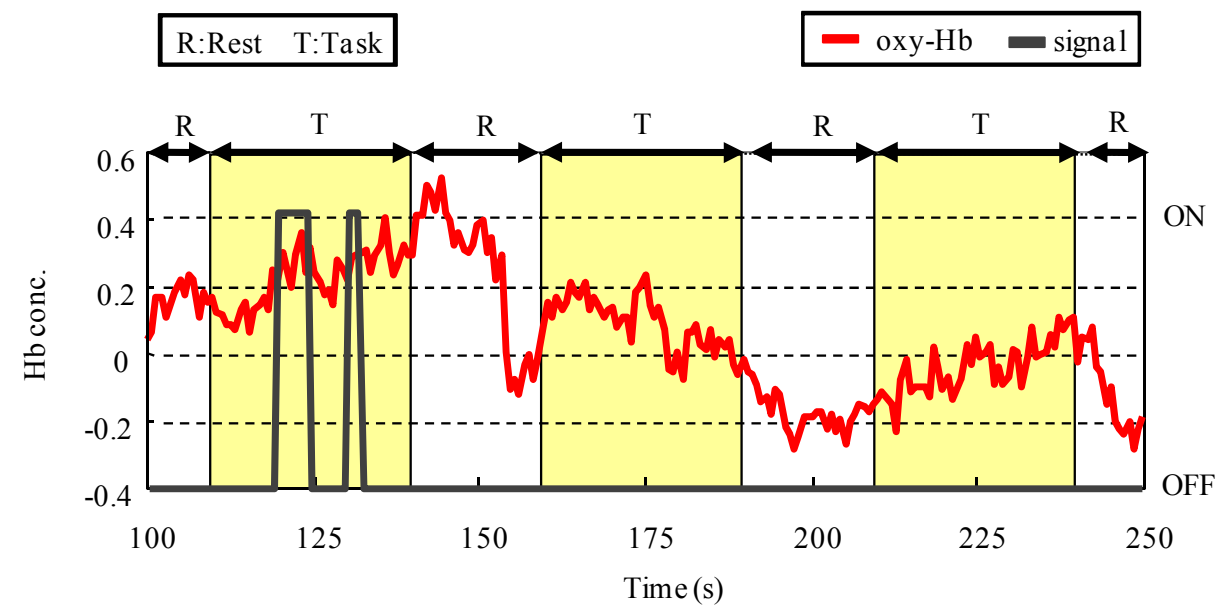

Fig. 14. Result of ON/OFF decision using conventional method

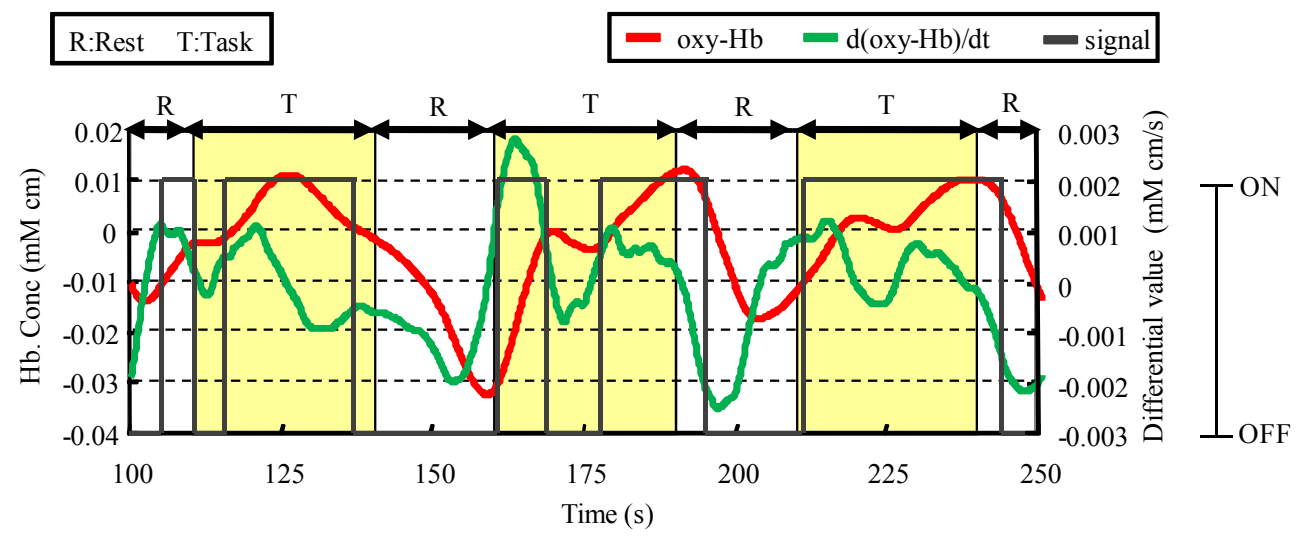

Fig. 15. Result of ON/OFF decision using proposed method (grasping task)

Some problems exist in the proposed detection method. For example, the ON detection was made during the rest time before starting the task because the differential had increased, and the OFF detection was made during the second task because the differential of oxy- $\mathrm{Hb}$ decreased during the rest time between tasks. However, the proposed detection method did enable highly accurate detection of many subjects, compared with the conventional detection method.

The detection of brain activity during imagined grasping tasks using the proposed detection method is presented in Fig. 16. Also during imagined grasping tasks, as in actual grasping tasks in Fig. 15, the ON detection was confirmed during all tasks. Furthermore, a correct detection could be made with little time delay, due to using the differential of oxy-Hb. These results confirm that the proposed detection method is valid for imagined grasping tasks as well. 


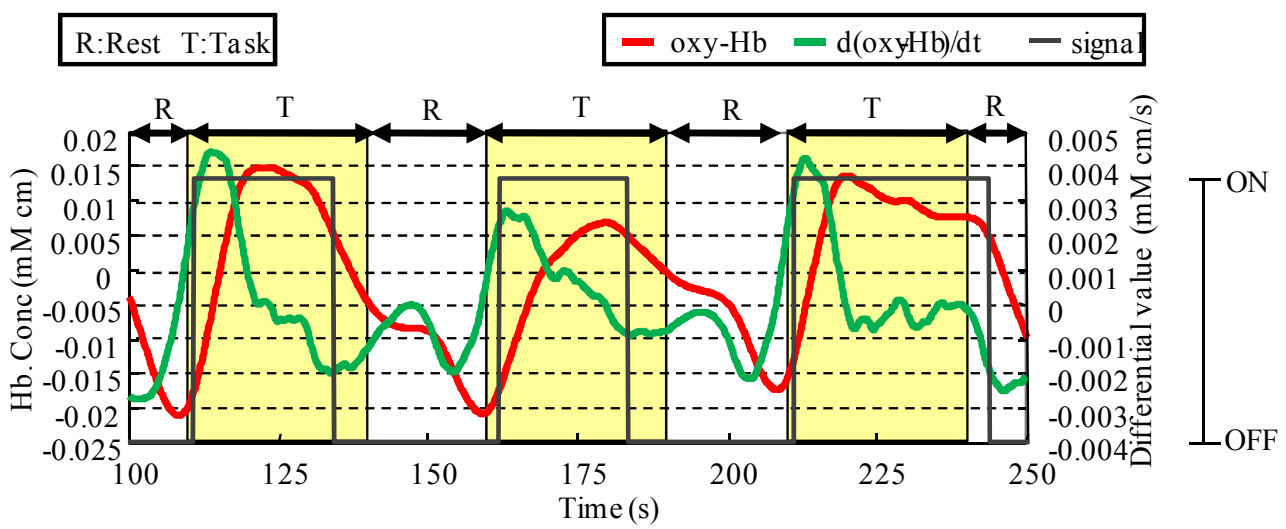

Fig. 16. Result of ON/OFF decision using proposed method (imagined grasping tasks)

\section{Summary}

In this study we proposed a new detection method that uses oxy-Hb and its differential as indexes for application to the NIRS-BCI rehabilitation system, detecting brain activity from the data measured using NIRS. First, we developed a BCI system to control robot arm using NIRS, and confirmed that NIRS-BCI system can control machine and device. When a detection method with a simple threshold is employed, no stable operation was made during the tasks. This study proposes a method by which oxy- $\mathrm{Hb}$ is plotted on the horizontal axis and the differential of oxy- $\mathrm{Hb}$ on the vertical axis, and brain activity is judged from the area of the plotted trajectory. As a result, we confirmed that the proposed detection method enables highly accurate detection with little time delay compared with the conventional detection method, during both grasping tasks and imagined grasping tasks.

Next, we applied NIRS-BCI system developed for rehabilitation. We also confirmed that the proposed detection method enables highly accurate detection compared with the detection method with a simple threshold during both grasping tasks and imagined grasping tasks. These results confirm the validity of the proposed detection method for the NIRS-BC rehabilitation system. We gathered experiment data from healthy men in the present study, but in future studies, we will develop portable NIRS-BCI rehabilitation system, and conduct experiments on patients with hemiplegia to put this system into practical use.

Furthermore, the detection method proposed in this study uses the oxy-Hb level and its differential alone as indexes, so it is applicable to the brain activity of the prefrontal area while the brain is performing cognition tasks, where deoxy-Hb exhibits various fluctuation patterns (Toichi M. et. al, 2004). Therefore, BCI can be expected to be applied to measure other than the motor area (e.g., the field of entertainment).

\section{Acknowledgment}

This work was supported by the Nihon University Multidisciplinary Research Grant in 2010 and 2011. 


\section{References}

Hochberg LR., Serruya MD., Friehs GM., Mukand JA., Seleh M., Caplan AH, Branner A., Chen D., Penn RD, Donoghue JP. (2006), "Neuronal ensemble control of prosthetic devices by a human with tetraplegia", Nature Vol.442 (7099), pp.164 -171

Pfurtscheller G., Leeb, R., Keinrath C., Friedman D., Neuper, C. (2006)“Walking from thought", Brain Res Vol.1071 (1), pp.145-152

Pfurtscheller G., Guger C., Müller G., Krausz G., Neuper C. (2000), “Brain oscillations control hand orthosis in a tetraplegic", Neurosci Lett Vol.292 (3), pp.211-214

Pfurtscheller G., Müller GR., Pfurtscheller J., Gerner HJ., Rupp R. (2003), “'Thought' control of functional electrical stimulation to restore hand grasp in a patient with tetraplegia", Neurosci Lett 351, pp.33-36

Vaughan TM, McFarland DJ, Schalk G, Sarnacki WA, Krusienski DJ, Sellers EW, Wolpaw JR. (2006), "The Wadsworth BCI Research and Development Program: at home with BCI", IEEE Trans Neural Syst Eng Vol.14 (2), pp.229 -233

Nagaoka T., Sakatani K, Awano T., Yokose N., Hoshino T., Murata Y., Katayama Y., Ishikawa A., Eda H. (2010), “Development of a new rehabilitation system based on brain-computer interface using near infrared spectroscopy," Advances in Experimental Medicine and Biology, Vol. 662, pp. 497-503

Jöbsis, F.F. (1977), "Non-invasive infrared monitoring of cerebral and myocardial oxygen sufficiency and circulatory parameters", Science, Vol. 198, pp. 1264-1267

Huettel, S. A. (2004). Functional Magnetic Resonance Imaging, Sinauer Associate, Inc., ISBN 978-0878932887, USA

Kojima T., Tsunashima H., Shiozawa T., Takada H., Sakai T. (2006), "Measurement of Train Driver's Brain Activity by Functional Near-Infrared Spectroscopy (fNIRS)," Computers in Railways X: Computer System Design and Operation in the Railway and Other Transit Systems, pp. 245-254

Yanagisawa K., Tsunashima H., Marumo Y., Hirose S., Shimizu T., Taira M., Haji T. (2009), "Measurement and evaluation of higher brain function by using functional nearinfrared spectroscopy (fNIRS)", Journal of Human Interface Society, Vol. 11(2) , pp. 183-191 (in Japanese)

Tsunashima H., Yanagisawa K. (2009), "Measurement of brain function of car driver using functional near-infrared spectroscopy (fNIRS)," Computational Intelligence and Neuroscience, Vol.2009

Elwell C.E., Springett R., Hillman E., Delpy D.T. (1999), “Oscillations in Cerebral Haemodynamics, Implications for Functional Activation Studies", Advances in Experimental Medicine and Biology, Vol. 471, pp. 57-65

Sakatani K., Murata Y., Fujiwara N., Hoshino T., Nakamura S., Kano T., Katayama Y. (2007), "Comparison of blood-oxygen-level-dependent functional magnetic resonance imaging and near-infrared spectroscopy recording during functional brain activation in patients with stroke and brain tumors," Journal of Biomedical Optics, Vol. 12 (6), p. 062110

Jueptner, M., Willer,C. (1995), “Dose measurement of regogional cerebral blood flow reflects synaptic activity?- implications for PET and fMRI," Neuroimage, Vol. 2, pp.148-156

Shimizu T., Hirose S., Obara H., Yanagisawa K., Tsunashima H., Marumo Y., Haji T., Taira M. (2009), "Measurement of frontal cortex brain activity attributable to the driving 
workload and increased attention," SAE International Journal of Passenger Cars Mechanical Systems, Vol. 2 (1), pp.736-744

Toichi M., Findling RL., Kubota Y., Calabrese JR., Wiznitzer M., McNamara NK., Yamamoto K. (2004), "Hemodynamic differences in the activation of the prefrontal cortex: attention vs. higher cognitive processing," Neuropsychologia, Vol. 42 (5), pp. 698-706 


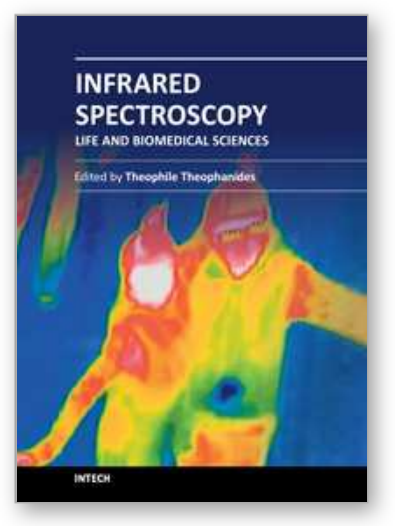

\author{
Infrared Spectroscopy - Life and Biomedical Sciences \\ Edited by Prof. Theophanides Theophile
}

ISBN 978-953-51-0538-1

Hard cover, 368 pages

Publisher InTech

Published online 25, April, 2012

Published in print edition April, 2012

This informative and state-of-the art book on Infrared Spectroscopy in Life sciences designed for researchers, academics as well as for those working in industry, agriculture and in pharmaceutical companies features 20 chapters of applications of MIRS and NIRS in brain activity and clinical research. It shows excellent FT-IR spectra of breast tissues, atheromatic plaques, human bones and projects assessment of haemodynamic activation in the cerebral cortex, brain oxygenation studies and many interesting insights from a medical perspective.

\title{
How to reference
}

In order to correctly reference this scholarly work, feel free to copy and paste the following:

Kazuki Yanagisawa, Hitoshi Tsunashima and Kaoru Sakatani (2012). Brain-Computer Interface Using NearInfrared Spectroscopy for Rehabilitation, Infrared Spectroscopy - Life and Biomedical Sciences, Prof. Theophanides Theophile (Ed.), ISBN: 978-953-51-0538-1, InTech, Available from: http://www.intechopen.com/books/infrared-spectroscopy-life-and-biomedical-sciences/brain-computerinterface-using-near-infrared-spectroscopy-for-rehabilitation

\section{INTECH}

open science | open minds

\section{InTech Europe}

University Campus STeP Ri

Slavka Krautzeka 83/A

51000 Rijeka, Croatia

Phone: +385 (51) 770447

Fax: +385 (51) 686166

www.intechopen.com

\section{InTech China}

Unit 405, Office Block, Hotel Equatorial Shanghai

No.65, Yan An Road (West), Shanghai, 200040, China 中国上海市延安西路65号上海国际贵都大饭店办公楼 405 单元

Phone: +86-21-62489820

Fax: $+86-21-62489821$ 
(C) 2012 The Author(s). Licensee IntechOpen. This is an open access article distributed under the terms of the Creative Commons Attribution 3.0 License, which permits unrestricted use, distribution, and reproduction in any medium, provided the original work is properly cited. 